MATEC Web of Conferences 12, 06001 (2014)

DOI: $10.1051 /$ matecconf $/ 20141206001$

(C) Owned by the authors, published by EDP Sciences, 2014

\title{
3D characterisation of RCF crack networks
}

\author{
Johan Ahlström, Casey Jessop, Lars Hammar and Christer Persson \\ Department of Materials and Manufacturing Technology, Chalmers University of Technology, \\ SE-412 96 Gothenburg, Sweden
}

\begin{abstract}
Rolling contact fatigue (RCF) damage is becoming more frequent with increased traffic and loading conditions in the railway industry. Defects which are characterized by a two-lobe darkened surface and a V-shaped surface-breaking crack are often so-called squats. The origination and propagation of squats in railway rails is the topic of many recent studies; the associated crack networks develop with complicated geometry near the surface of rails that is difficult to characterise using most non-destructive methods. The cracks can be examined with repeated metallographic sectioning, but the process is time-consuming and destructive. In order to reduce time, as well as information and material loss, high-resolution and high-energy X-ray imaging of railway rails was done in the current study. Combining the exposures from a range of angles using image analysis, a 3D representation of the complex crack network is achieved. The latter was complemented with metallographic sectioning to determine the accuracy of prediction of the geometrical reconstruction.
\end{abstract}

\section{Introduction}

Heavier loads and increased accelerations and speeds on today's railways are causing increasing amounts of damage to rails and wheels. The early detection of rail surface/near-surface defects could help reduce the frequency and intensity of failures and accidents. In order to develop a proper maintenance system, it is important to firstly understand the nature, cause, and consequences of different defect types. In recent years, so-called squats have been the subject of several investigations and are proving to be an important cause of failure worldwide.

Squats are a particular type of defects that generally manifest themselves by a two-lobed dark spot on the rail surface and a planar crack network parallel to the surface, within a shallow depth [1]; however, in some cases they may develop cracks perpendicular to the surface, causing rail transverse fracture. By the time the crack network can be detected using ultrasonic inspection or Eddy current testing, it could have traveled at least $4 \mathrm{~mm}$ into the rail, making it a severe squat [2]. Although on-site non-destructive detection systems could be used to find squats, these methods cannot visualize the 3D geometry of the crack network and thus not judge the severity of the damage.

A radiography study was performed on rail sections taken from field with suspected squat-like defects, in order to observe the network of cracks associated to squats and attempting to reconstruct its 3D shape - at present only achievable by metallographic cross-sectioning. Compared to metallographic techniques, the use of X-ray is more beneficial for the study of squats; it requires less time, less

This is an Open Access article distributed under the terms of the Creative Commons Attribution License 4.0, which permits unrestricted use, distribution, and reproduction in any medium, provided the original work is properly cited. 


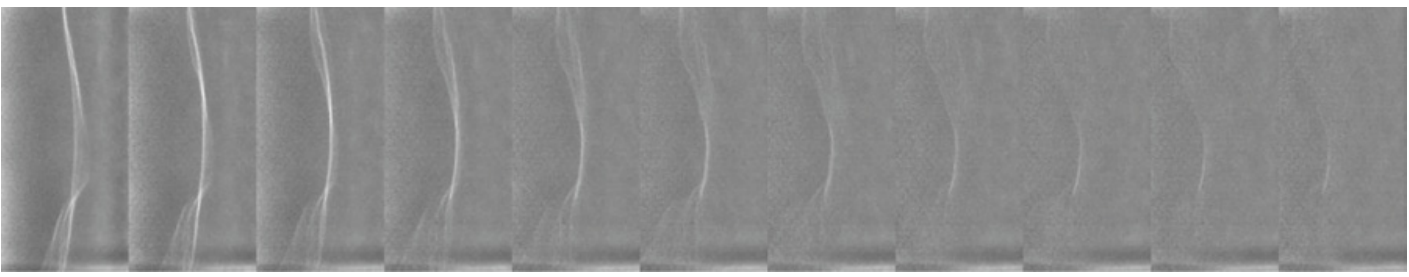

Figure 1. Series of pictures exposed at different angles from $+80-+60$ degrees relative to the rail head normal.

manpower, no destruction of sample and no evidence loss. If the method can be applied in field it can be used to judge the severity of damage and be used for maintenance planning. This paper also aims at revealing the potential of this method in other fields, as a novel NDT method.

\section{Experimental}

A piece of UIC60 rail containing four squats generated under service loading was received. The rail head was cut off and placed vertically on a rotation table in between the X-ray source and the detector. Reference position indicators were mounted on the surface of the rail head at known positions. Exposures were made at different angles, with 2 degrees step, in ranges where the cracks were anticipated to be oriented parallel with the beam. Images were stored and mathematically treated for improved contrast. The different images were analyzed to extract the necessary geometrical information for $3 \mathrm{D}$ reconstruction. A detailed description of the experimental setup and similar techniques for reconstruction are reported elsewhere [3]. Thereafter the rail head was cut at known positions along the rail, perpendicular to the rail centerline. Each cut was carefully prepared by metallographic methods, not to cause metal flow over closed cracks. The crack network was measured and stored. The reconstructions done by radiographic and metallographic techniques were compared.

\section{Example of results}

Figure 1 shows a compiled picture of one squat showing the different angles of exposure from $+80-$ +60 degrees relative to the rail head normal. It is evident that the relation between the angle of exposure and the crack plane is critical for the visibility of the crack.

\section{Discussion}

The well-established tomography techniques with complete 360 degree scanning and following digital volumetric reconstruction, requires little knowledge of the crack geometry beforehand. However, in a rail head in field, certain angles of exposure are out of reach. Thus, rotating radiography technique needs to be used instead. In this technique, one utilizes the characteristic of radiography to be sensitive to alignment between the beam and the crack. This helps sorting out the normal of the crack plane in each picture. Putting together information on shape, crack plane orientation, and movement relative to the reference position indicators, and comparing between different angles of exposure, $3 \mathrm{D}$ reconstruction is possible.

Closer to the crack tip the crack opening can be too narrow for detection with the given resolution. Thus, information from metallographic sectioning, and characterization of different crack types is necessary to verify the results of radiographic techniques. 


\section{References}

[1] A. S. S. Simon, C. Dayot, X. Quost, Y. Berthier, "Tribological characterization of rail squat defects," Wear 297, pp. 926-942, 2013

[2] C. R. M. Ph. Papaelias, C.L. Lewis, "A review on non-destructive evaluation of rails: state-of-theart and future development," Journal of Rail and Rapid Transit 222, pp. 367-384, 2008

[3] E. Lindgren and L. Hammar, Evaluation of an automatic system for detection and positioning of small pore defects using digital radiography, AIP Conference Proceedings 1511, 1646 (2013); doi: $10.1063 / 1.4789239$ 